\title{
PÂTURAGE EN ROTATION CHEZ BRACHIARIA BRIZANTHA CV. MARANDU POUR AMÉLIORER LA PRODUCTION DE LAIT
}

\section{ARTICLE ORIGINAL}

SOARES, Marcio Groto ${ }^{1}$, CAMARGO, Simone Cristina², FRASSETTO, Matheus Orlandin ${ }^{3}, \mathrm{ABREU}$, Hélio Mar de ${ }^{4}$

SOARES, Marcio Groto. Et al. Pâturage en rotation chez Brachiaria brizantha cv. marandu pour améliorer la production de lait. Revista Científica Multidisciplinar Núcleo do Conhecimento. Année. 06, Ed. 09, Vol. 07, p. 104 à 118. Septembre 2021. ISSN : 2448-0959, Lien d'accès: https://www.nucleodoconhecimento.com.br/veterinaria-fr/paturage-en-rotation, DOI: 10.32749/nucleodoconhecimento.com.br/veterinaria-fr/paturage-en-rotation

\section{RÉSUMÉ}

Dans les propriétés laitières, pour qu'il y ait augmentation du profit, il faut augmenter l'efficacité des facteurs de production, notamment la nutrition, qui impacte le coût fixe. De plus en plus, un équilibre est recherché entre l'approvisionnement en fourrage et en pâturage. La méthode de pâturage en rotation a été adoptée par plusieurs producteurs, car elle a une bonne productivité dans une zone plus petite. Cependant, les pâturages utilisés dans ce type de pâturage, malgré leur excellente qualité, ont des besoins nutritionnels plus élevés. L'objectif de ce travail était de réaliser une analyse économique du coût de la production laitière, à travers l'implantation de pâturages en rotation, et l'échange de pâturage indigène contre Brachiaria brizantha $c v$. marandu, sur une ferme familiale. Ce travail a été préparé dans la ville de Foz do Iguaçu. Une superficie de $6300 \mathrm{~m}^{2}$ a été utilisée, divisée en 28

\footnotetext{
${ }^{1}$ Diplômé de l'université.

2 Doctorat.

${ }^{3}$ Prof.

${ }^{4}$ Diplômé de l'université.
}

RC: 99521

Disponible en: https://www.nucleodoconhecimento.com.br/veterinaria-fr/paturage-enrotation 
paddocks de $225 \mathrm{~m}^{2}$ à l'aide d'une clôture électrique. Le coût total de production était de $6047,40 \mathrm{R} \$$, avec une marge positive de 5,143,61 $\mathrm{R} \$$ par an. D'après les résultats obtenus dans les analyses économiques, il est conclu que l'implantation du pâturage en rotation et l'échange de pâturage indigène contre Brachiaria brizantha $c v$. Marandu est une gestion viable, puisque les revenus générés par la vente du lait étaient suffisants pour couvrir les coûts totaux de production.

Mots-clés: Coût, Lait, Fourrage, Piquet, Manutention.

\section{INTRODUCTION}

Le Brésil se classe au quatrième rang mondial de la production laitière. En 2018, la région sud représentait $34 \%$ de la production totale du pays (ANUÁRIO LEITE, 2019). L'État du Paraná à lui seul a produit cette année en moyenne 4,37 milliards de litres de lait, dont $19 \%$ dans l'ouest du Paraná (IBGE, 2018).

Les bovins laitiers dans l'État du Paraná sont une activité typique de l'agriculture familiale, représentant environ $85,1 \%$ des producteurs de l'État (SEAB, 2018). La plupart de ces producteurs utilisent les pâturages comme principale source de nourriture pour les animaux. La superficie moyenne occupée par les pâturages dans l'État est de 16,6 hectares (IPARDES, 2009).

Bien que le Brésil se distingue par sa production laitière, sa productivité est d'environ 1689 L/vache/an, ce qui est inférieur à celui des États-Unis, de la Chine, de la Russie, de l'Argentine, de I'Union européenne et de la Nouvelle-Zélande, qui produisent plus de $3 \mathrm{~L} / v a c h e / a n$. L'un des facteurs responsables du faible rendement laitier est l'utilisation d'herbes peu productives et de faible qualité nutritionnelle, ainsi qu'une gestion inadéquate des animaux et des pâturages (GONÇALVES et al., 2003).

Une façon d'intensifier la production animale dans les systèmes de pâturage est d'adopter un fourrage amélioré, plus adapté au climat, avec un potentiel de

RC: 99521

Disponible en: https://www.nucleodoconhecimento.com.br/veterinaria-fr/paturage-enrotation 
production plus élevé et de meilleure qualité (JANK, 2017). Parmi les différents fourrages existant au Brésil, se distingue la variété hybride d'herbe brachiaria brizantha $c v$. marandu, pour présenter une bonne valeur nutritionnelle et une production élevée de biomasse, s'adapter bien aux sols de fertilité moyenne et bonne, être tolérant à l'acidité du sol (FONSECA et al., 2010).

La gestion correcte des pâturages devrait être un autre point à observer dans les propriétés des bovins laitiers. L'un des pâturages qui s'est avéré viable est la rotation, où il y a subdivision du pâturage en trois piquets ou plus. Ce type de pâturage présente plusieurs avantages, tels que: une plus grande uniformité du pâturage, une plus grande utilisation de fourrages, un taux de stockage plus élevé et une longévité plus longue des herbes qui forment des touffes (FUKUMOTO et al., 2010).

Différentes études ont montré de bons résultats pour les bovins laitiers, avec l'implantation d'un pâturage rotatif dans l'herbe Brachiaria brizantha $c v$. marandu (GONÇALVES, 2003); (DEMSKI, 2013), (MOURA, 2017). Selon (ANDRADE, 2008), ce type de pâturage ne tolère pas le pâturage continu.

Avec le bon choix du fourrage, une bonne gestion et une meilleure utilisation de la superficie de la propriété, il cherche à générer des économies pour le producteur, car il réduira le coût de production. Selon (GERON, 2012), le coût de production est la somme de tous les montants dépensés pour les opérations et les services d'insumand utilisés dans le processus de production d'une certaine activité. Avec l'étude des coûts, il est possible de vérifier comment les ressources utilisées dans un processus de production sont rémunérées et comment est la rentabilité de l'activité (GERON et al., 2014).

Ainsi, l'objectif de ce travail était d'effectuer l'analyse économique du coût de la production laitière, à travers la mise en œuvre du pâturage en rotation, et l'échange

RC: 99521

Disponible en: https://www.nucleodoconhecimento.com.br/veterinaria-fr/paturage-enrotation 
de pâturages indigènes contre des pâturages de Brachiaria brizantha cv. marandu, sur un domaine agricole familial.

\section{MATÉRIEL ET MÉTHODES}

L'expérience a été réalisée dans une propriété laitière située dans la ville de Foz do Iguaçu - PR, Latitude: -25.5469, Longitude: -54.5882 25 32' 49 » Sud, 54 $35^{\prime} 18$ » Ouest. Cette propriété est composée d'une superficie de 5,0 hectares, dans cette zone a été développé un bovin laitier traditionnel à la région, où les animaux avaient accès à toute la zone de pâturage, composée d'herbe indigène de faible productivité, il n'y avait pas de disponibilité de sel minéral, seulement du sel commun dans l'auge et n'étant disponible qu'une supplémentation au moment de la traite de $10 \mathrm{~kg}$ de par les animaux en lactation et l'accès à l'eau était à partir d'un ruisseau qui traverse la propriété.

La propriété compte six bovins laitiers, composés de quatre vaches adultes, deux vaches allaitantes et deux vaches séchées, plus deux génisses.Ces animaux ont été gérés dans toute la zone de pâturage tout au long de l'année et les animaux en lactation ont reçu $10 \mathrm{~kg}$ d'aliments industrialisés par jour.

Les travaux ont commencé par une visite à la propriété pour un diagnostic. Les mesures de la superficie des pâturages ont été effectuées à l'aide d'un GPS (figure 1) et de la collecte du sol (figure 2). Les résultats de l'analyse du sol sont (tableau 1).

RC: 99521

Disponible en: https://www.nucleodoconhecimento.com.br/veterinaria-fr/paturage-enrotation 
Figure 1 - Photo satellite de la zone expérimentale, avec la démarcation de la zone de pâturage:

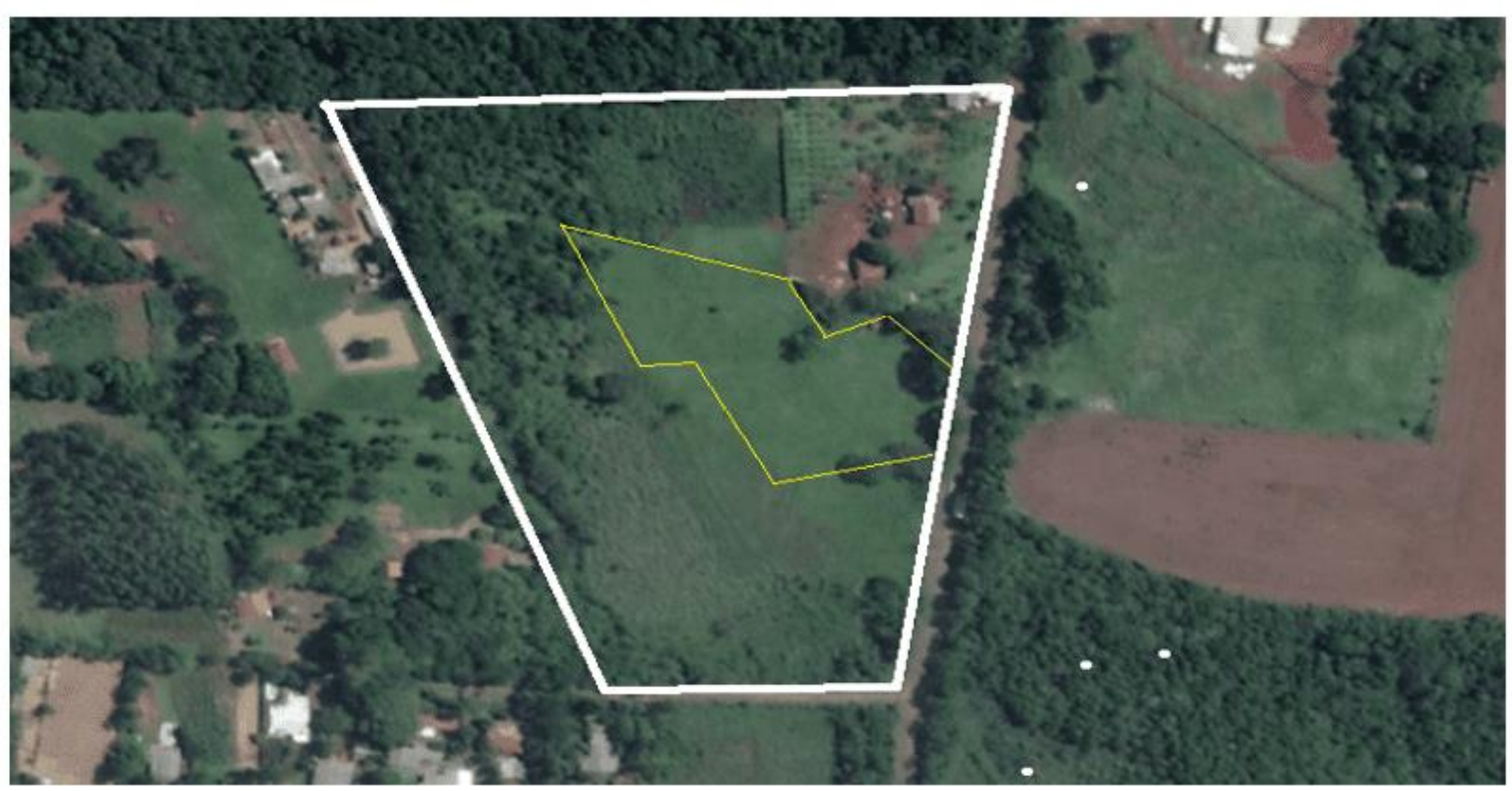

Source : Google Earth.

Figure 2 - Prélèvement d'échantillons de sol:

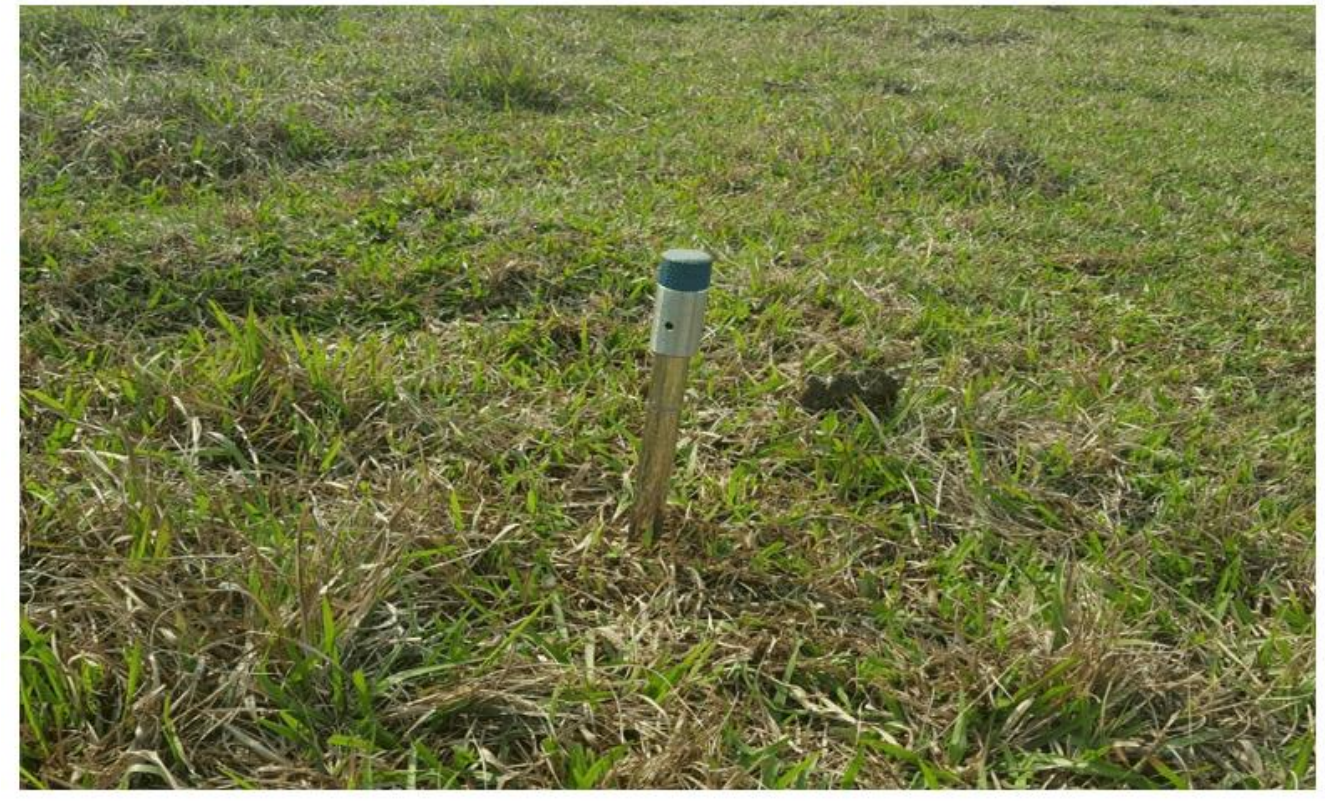

Source : Archives personnelles.

RC: 99521

Disponible en: https://www.nucleodoconhecimento.com.br/veterinaria-fr/paturage-enrotation 
Tableau 1 - Résultats de l'analyse du sol de la zone expérimentale:

\begin{tabular}{|c|c|c|c|c|c|c|c|c|c|c|c|c|c|c|}
\hline $\mathrm{pH}$ & & & $\mathrm{K}$ & $\mathrm{Ca}$ & $\mathrm{Mg}$ & $\mathrm{Al}$ & $\mathrm{H}+\mathrm{Al}$ & CTC & SB & $\mathrm{V}$ & $\mathrm{m}$ & $\mathrm{Ca}$ & $\mathrm{Mg}$ & $\mathrm{K}$ \\
\hline \multirow[t]{2}{*}{$\mathrm{CaCl} 2$} & M.O & $\mathrm{P}$ (res) & & & & & & & & & & & & \\
\hline & $\mathrm{g} / \mathrm{Kg}$ & $\mathrm{mg} / \mathrm{dm} 3$ & \multicolumn{5}{|c|}{$\mathrm{mmol} / \mathrm{dm} 3$} & $\%$ & & & \multicolumn{4}{|c|}{$\%$ СТС } \\
\hline 5,2 & 38 & 14 & 3 & 21 & 5 & 2,7 & 29 & 58 & 29 & 50 & 8 & 36 & 9 & 5 \\
\hline
\end{tabular}

Source : FZEA/USP.

Après avoir reçu l'analyse du sol, des calculs de correction du sol ont été effectués, où le $\mathrm{V} \%$ était de $58 \%$. Pour corriger le $\mathrm{pH}$ du sol, $800 \mathrm{~kg} \mathrm{ha}^{-1}$ de calcaire dolomitique à $86 \%$ de PRNT ont été nécessaires. Pour corriger le phosphore, il a fallu ajouter $250 \mathrm{~kg} \mathrm{ha}^{-1}$ d'engrais simple superphosphate. Pour la fertilisation de la production, $250 \mathrm{~kg} \mathrm{ha}^{-1}$ d'urée agricole ont été utilisés (FRASSETO, 2015).

Pour commencer la préparation du sol, l'application de calcaire a été appliquée dans toute la zone expérimentale et, peu de temps après, une grille lourde a été utilisée, d'une profondeur de $40 \mathrm{~cm}$, qui a servi à incorporer du calcaire dans le sol, à briser les compactages de surface et à donner des conditions à la croissance des nouvelles espèces fourragères.

Pour un dimensionnement de la surface nécessaire au pâturage, les vaches ont été pesées à l'aide d'un ruban de pesée avec une précision de 95\%, totalisant $1950 \mathrm{~kg}$ ou 4,5 UA. Étant donné que des bovins laitiers sont utilisés, il a été déterminé que les animaux auraient accès à un nouveau paddock à la fin d'une journée de pâturage. Par conséquent, les données de (FRASSETO 2015) ont été utilisées, dans lesquelles une UA (450 kg de poids vif) a besoin d'au moins $50 \mathrm{~m}^{2}$ par jour de zone de pâturage. Sachant qu'il y avait 4,5 UA, la superficie de chaque paddock était de $225 \mathrm{~m}^{2}$. Au total, 28 paddocks ont été construits, sur 0,63 hectare. Lors de la construction des piquets, il a fallu ajouter trois couloirs de $2 \mathrm{~m}$ de large.

RC: 99521

Disponible en: https://www.nucleodoconhecimento.com.br/veterinaria-fr/paturage-enrotation 
Pour le fractionnement des piquets ont été utilisés 50 mourões, 72 barres d'armature de $10 \mathrm{~mm}, 32$ barres d'armature de $12 \mathrm{~mm}, 500 \mathrm{~m}$ de fil tri galvanisé pour environ le périmètre total de la zone et $2500 \mathrm{~m}$ de fil électroplastique pour la division interne des piquets, 150 isolateurs de type brun, 22 isolateurs de crochet et 114 isolateurs de type barres d'armature. Un fil métallique d'une hauteur de $80 \mathrm{~cm}$ a été utilisé par rapport au sol et chaque $10 \mathrm{~m}$, une barre d'armature de $1,5 \mathrm{~m}$ avec isolant a été implantée pour maintenir la hauteur par rapport au sol, du fil électroplastique a également été utilisé pour diviser les piquets et réduire les coûts de formation. Pour électrifier le fil, un électrificateur Sentinel modèle 30000 a été utilisé, avec un minimum de 5000 volts.

Figure 3 - Croquis de la division du piquetage :

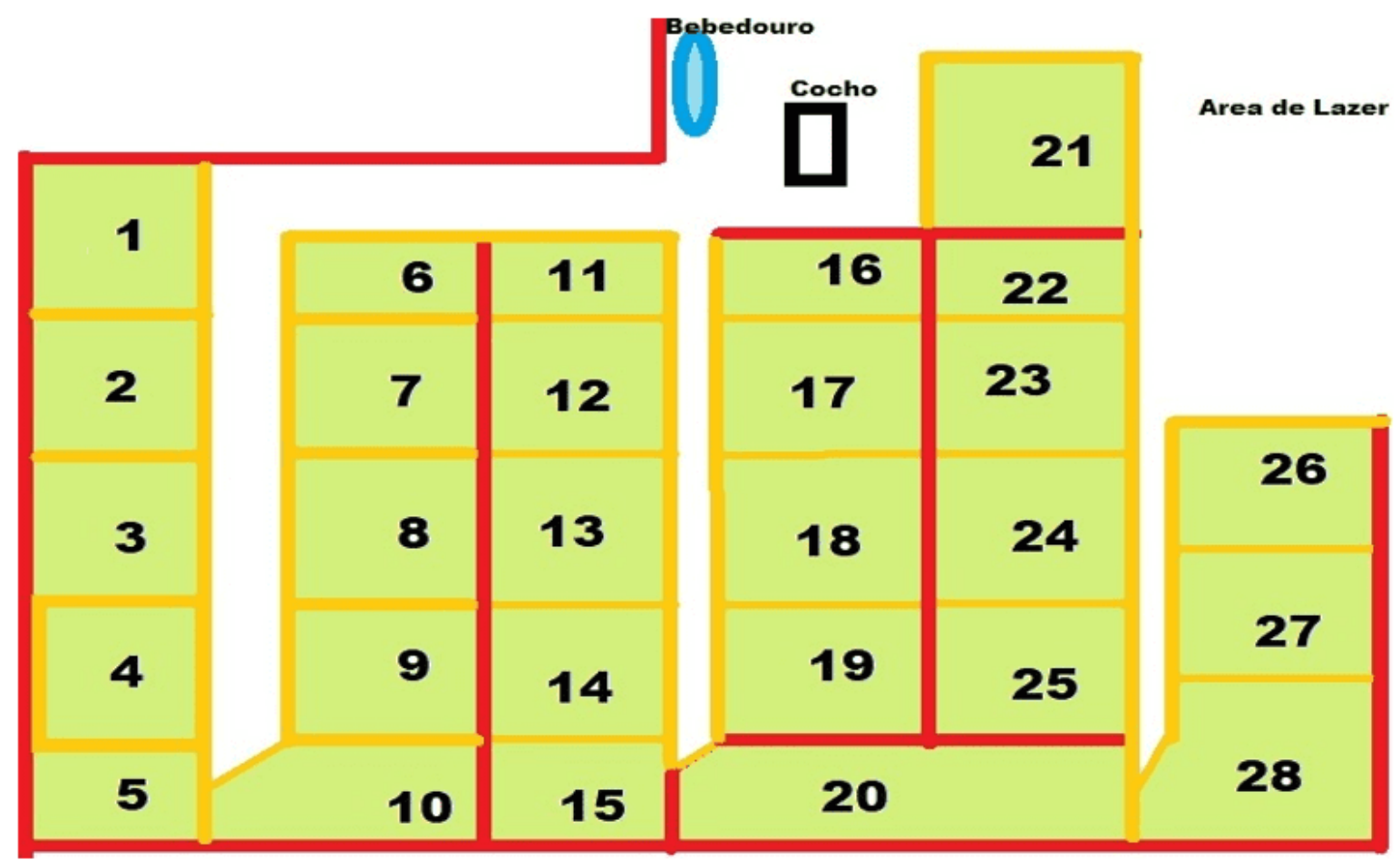

Source : Archives personnelles.

Le fourrage choisi était Brachiaria Brizantha cv. marandu, la gestion de ce fourrage commence lorsque la plante atteint $30 \mathrm{~cm}$ en entrée et $15 \mathrm{~cm}$ en sortie Andrade

RC: 99521

Disponible en: https://www.nucleodoconhecimento.com.br/veterinaria-fr/paturage-enrotation 
(2008) ; Fonseca (2010). La plantation de fourrage et la fertilisation de la plantation ont commencé le 15/11/2019, avec la méthode de plantation à la volée et l'incorporation de graines et d'engrais phosphatés super simples par la suite. Les graines ont été incrustées, en utilisant $15 \mathrm{~kg} \mathrm{ha}^{-1}$.

La première fertilisation de production a été réalisée 60 jours après la plantation, en appliquant $125 \mathrm{~kg} \mathrm{ha}^{-1}$ d'urée agricole à $45 \%$ d'azote. Le premier pâturage a commencé le 02/03/2020, 77 jours après la plantation. La deuxième fertilisation de la production étant appliquée $125 \mathrm{Kg} \mathrm{ha}^{-1}$ d'urée agricole a été réalisée dans le premier cycle de pâturage soit 28 jours après le début du premier pâturage.

Pour le reste des animaux a été ajouté un buveur d'eau avec environ $100 \mathrm{~L}$ d'eau, l'ajout de sel minéral adapté aux bovins laitiers et les arbres de la propriété euxmêmes ont servi d'ombrage.

Pour effectuer l'analyse économique et l'évaluation du coût de production laitière, nous avons identifié les agents impliqués, ainsi que les valeurs qui représentent les effets de la mise en œuvre du projet sur ces agents, en tenant également compte de la clôture d'un an.

Pour évaluer le coût de mise en œuvre du projet, nous avons examiné les coûts d'investissement dans la préparation du sol et la plantation de l'herbe, ainsi que les investissements dans l'infrastructure et l'équipement dans la formation des piquets.

Pour évaluer le coût de production, nous avons utilisé la structure du coût d'exploitation de la production, proposée par Matsunaga (1976). Dans lequel, le coût opérationnel effectif (COE), correspond à la dépense d'argent par le producteur, avec l'achat d'aliments, les dépenses avec l'entretien des pâturages et des médicaments, pendant un an.

Pour le coût total de production (CTP), l'amortissement a été ajouté à (COE). La dépréciation annuelle du pâturage a été calculée par la somme des fournitures

RC: 99521

Disponible en: https://www.nucleodoconhecimento.com.br/veterinaria-fr/paturage-enrotation 
nécessaires à la construction de la clôture électrique répartie sur une période de dix ans. Amortissement de $10 \%$ par année (CANZIANI et al., 2000).

Pour calculer les recettes, on a tenu compte des recettes nettes, c'est-à-dire du montant gagné en livraison de lait par année moins les coûts de production totaux (MARTIN, 1997).

\section{RÉSULTATS ET DISCUSSION}

Dans la production agricole, il est nécessaire d'avoir une planification par le producteur, afin qu'il y ait une croissance et un développement de l'activité. Par conséquent, la première étape de l'étude de faisabilité d'un projet consiste à étudier les dépenses pour sa mise en œuvre. Les coûts de mise en œuvre de ce projet, de la préparation du sol pour la plantation du pâturage, à la formation des piquets de grève étaient de $3023,70 R \$$.

Avant d'effectuer l'implantation de l'herbe $B$. brizantha cv. marandu, il fallait préparer le sol. Les investissements liés au travail du sol sont décrits dans le tableau 2.

Tableau 2 - Coûts d'investissement dans la préparation du sol et la plantation :

\begin{tabular}{|l|l|l|l|}
\hline Description & Unité & Quantité & Valeur (R\$) \\
\hline Analyse des sols & Uni & 1 & 33,00 \\
\hline $\begin{array}{l}\text { Calcaire dolomitique (PRNT } \\
\text { 86\%) }\end{array}$ & $\mathrm{Kg}$ & 800 & 156,00 \\
\hline $\begin{array}{l}\text { Superphosphate (19.00.00) } \\
\text { Temps d'aération du tracteur }\end{array}$ & $\mathrm{Kg}$ & 250 & 287,46 \\
\hline Graines incrustées & $\mathrm{K}$ & 2 & 400,00 \\
\hline Urée agricole & $\mathrm{Kg}$ & 15 & 287,01 \\
\hline Herbicide Glyphosate & $\mathrm{Ml}$ & 500 & 300,00 \\
\hline Huile 2T & $\mathrm{Ml}$ & 250 & 20,00 \\
\hline
\end{tabular}

RC: 99521

Disponible en: https://www.nucleodoconhecimento.com.br/veterinaria-fr/paturage-enrotation 


\begin{tabular}{|l|l|l|l|}
\hline Carburant - Essence & L & 5 & 20,00 \\
\hline Total & & & $\mathbf{1 . 6 3 8 , 4 7}$ \\
\hline
\end{tabular}

Source : Données du projet.

Les coûts du travail du sol et de la plantation représentaient 54,20 \% des coûts totaux d'implantation des pâturages. Les pratiques correctives et la fertilisation des sols ne sont qu'une partie des exigences nécessaires au succès du système de production des pâturages (PEREIRA et al., 2018).

II est important pour le producteur d'investir et d'effectuer une bonne préparation du sol et une mise en œuvre correcte du pâturage, en recherchant toujours l'équilibre dans le système sol-plante-animal (PEREIRA et al., 2018). Ces gestions influenceront l'augmentation de la production animale, ainsi que la réduction des dépenses alimentaires pour l'alimentation animale.

Dans la recherche d'une gestion correcte du pâturage, il a été subdivisé en piquets. Les investissements dans l'infrastructure et l'équipement dans la formation des piquets de grève sont représentés dans le tableau 3 .

Tableau 3 - Tableau des coûts pour la formation des piquets de grève, avec la clôture électrique:

\begin{tabular}{|l|l|l|l|}
\hline Description & Unité & Quantité & Valeur (R\$) \\
\hline Fil Eletrix & $\mathrm{m}$ & 500 & 184,00 \\
\hline Fil électroplastique 500 m & $\mathrm{m}$ & 5 & 104,82 \\
\hline Isolant brun & Uni & 150 & 176,98 \\
\hline Isolant d'armature & Uni & 114 & 131,65 \\
\hline Isolant de type crochet court & Uni & 20 & 24,00 \\
\hline Isolant à crochet long & Un & 2 & 4,60 \\
\hline Commutateur de commutation & Un & 3 & 36,00 \\
\hline
\end{tabular}

RC: 99521

Disponible en: https://www.nucleodoconhecimento.com.br/veterinaria-fr/paturage-enrotation 


\begin{tabular}{|c|c|c|c|}
\hline $\begin{array}{l}\text { Barres d'armature }(3 / 8 ») \text { 1,5 } \\
m\end{array}$ & Un & 72 & 284,30 \\
\hline $\begin{array}{l}\text { Barres d'armature }(1 / 2 ») \text { 1,5 } \\
m\end{array}$ & Un & 2 & 186,18 \\
\hline Tube PVC $20 \mathrm{~mm} 6 \mathrm{~m}$ & Un & 1 & 12,80 \\
\hline Voltmètre numérique & Un & 1 & 139,90 \\
\hline Électrifier & Un & 1 & 100,00 \\
\hline Total & & & $1.385,23$ \\
\hline
\end{tabular}

Source : Données du projet.

Les coûts d'infrastructure et d'équipement pour la formation des piquets représentent $45,80 \%$ des coûts totaux de mise en œuvre du pâturage. La gestion rationnelle des fourrages a amplement démontré les avantages de la division des pâturages, où il y a une plus grande uniformité de pâturage, une plus grande utilisation des fourrages, un taux de charge plus élevé et une plus grande longévité des graminées qui forment des touffes (FUKUMOTO, 2010), ce qui est le cas de B. brizantha cv. marandou. L'utilisation de la clôture électrique, sous ses différentes formes en piquetage, est un outil nécessaire pour que les lotissements que nécessite le pâturage en rotation puissent être mis en œuvre à faible coût (EMBRAPA, 1999).

Les actifs qui composent l'entreprise sont soumis à des dévaluations constantes, principalement en raison de l'usure et du vieillissement. (Tableau 4) contient les données d'amortissement de la clôture électrique.

Tableau 4 - Amortissement des matériaux et de l'équipement :

\begin{tabular}{|l|l|l|l|}
\hline Description & $\begin{array}{l}\text { Montant total } \\
\mathrm{R} \$\end{array}$ & $\begin{array}{l}\text { Durée de conservation } \\
\text { (années) }\end{array}$ & $\begin{array}{l}\text { Valeur } \\
\text { annuelle }\end{array}$ \\
\hline
\end{tabular}

RC: 99521

Disponible en: https://www.nucleodoconhecimento.com.br/veterinaria-fr/paturage-enrotation 
Construction de clôture 1.385,25

électrique

Source : Données du projet.

Tableau 5 - Paiement des placements:

\begin{tabular}{|l|lr|l|l|l|}
\hline Description & $\begin{array}{l}\text { Montant } \\
\mathrm{R} \$\end{array}$ & total & $\begin{array}{l}\text { Délai de paiement } \\
\text { (années) }\end{array}$ & $\begin{array}{l}\text { Valeur } \\
\text { annuelle }\end{array}$ \\
\hline $\begin{array}{l}\text { Coût } \\
\text { déploiement }\end{array}$ & $\mathbf{3 , 0 2 3 . 7 0}$ & $\mathbf{1 0}$ & 302.37 \\
\hline
\end{tabular}

Source : Données du projet.

Pour avoir un plus grand contrôle annuel sur la gestion de l'entreprise, les investissements pour le déploiement ont été divisés en 10 ans. 4,20 \% des revenus annuels provenant de la vente de lait, décrits dans le tableau 5.

Pour calculer l'apport monétaire, sous forme d'argent en un an, on considérait le montant versé au producteur par litre de lait, vendu quotidiennement et directement du producteur de formulaire pendant un an. Pour calculer la quantité de lait annuelle, on considérait une traite quotidienne le matin avec un total de 10 litres / jour (tableau $6)$.

Tableau 6 - Recettes provenant de la vente de lait annuel :

\begin{tabular}{|l|l|}
\hline Variables & Valeur totale \\
\hline Prix par litre $(\mathrm{R} \$)$ & 2,00 \\
\hline $\begin{array}{l}\text { Litres totaux de lait produits } \\
(\mathrm{L})\end{array}$ & $3.600,00$ \\
\hline
\end{tabular}

RC: 99521

Disponible en: https://www.nucleodoconhecimento.com.br/veterinaria-fr/paturage-enrotation 
Recettes finales $(\mathrm{R} \$)$

Source : Données du projet.

Chaque année, le producteur devra effectuer une dépense d'argent, qui est considérée comme un coût opérationnel effectif (COE). Les coûts des aliments pour animaux, les dépenses d'entretien des pâturages et les médicaments ont été pris en compte (tableau 7).

Tableau 7 - Coûts d'exploitation effectifs - COE, par année :

\begin{tabular}{|l|l|l|l|l|}
\hline Description & Unité & Quant & Valeur unitaire R\$ & Montant total R\$ \\
\hline Ration & $\mathrm{Kg}$ & 720 & 1,32 & 950,04 \\
\hline Urée agricole & $\mathrm{Kg}$ & 250 & 1,60 & 400,00 \\
\hline Énergie électrique & $\mathrm{R} \$$ & 12 & 0,60 & 216,00 \\
\hline $\begin{array}{l}\text { Vaccin contre } \\
\text { brucellose }\end{array}$ & Dose & 6 & 1,62 & 9,72 \\
\hline Vaccin contre la rage & Dose & 6 & 1,48 & \\
\hline Dectomax 50 mL & Uni. & 1 & 21,90 & 21,90 \\
\hline Vaccin antiphteuse & Dose & 6 & 1,50 & 9,00 \\
\hline Total & & & & $\mathbf{1 . 6 1 5 , 5 0}$ \\
\hline
\end{tabular}

Source : Données du projet.

Il est observé dans ce travail que les dépenses de production les plus importantes sont consacrées aux aliments pour animaux. Selon (MARTINEZ, 2009), les dépenses en nutrition représentent en moyenne $67 \%$ des coûts de production du lait au Brésil. Ainsi, des stratégies sont recherchées pour réduire l'utilisation de régimes et d'autres suppléments. L'amélioration et la gestion des pâturages sont une alternative qui aide la production laitière à être économiquement viable. Pour cette raison, le pâturage en rotation a été de plus en plus indiqué (MARION et al., 2010).

RC: 99521

Disponible en: https://www.nucleodoconhecimento.com.br/veterinaria-fr/paturage-enrotation 
Rosestolato (2015), en analysant la viabilité économique de la production laitière de deux propriétés distinctes en ce qui concerne le système de production technologique, a observé que le (COE) sur la propriété dans laquelle les vaches étaient gardées dans un système de pâturage continu et complété par des aliments concentrés et du sel minéral était plus élevé que celui maintenu dans un système de pâturage rotatif et complété par de la canne à sucre hachée, aliments concentrés et sel minéral.

Dans la propriété évaluée dans cette étude, $10 \mathrm{~kg}$ d'aliments industrialisés ont été donnés par animal en lactation et après la mise en œuvre du projet, il y a eu une diminution de $9 \mathrm{~kg}$ commençant à n'offrir que $1 \mathrm{~kg}$ d'aliments aux animaux, diminuant ainsi le (COE), comme observé par Rosestolato (2015). Cependant, la supplémentation en animaux, même avec le changement du système de pâturage, est importante, car elle aide à fournir aux animaux les nutriments qui manquent dans la composition du fourrage. Le coût de l'aliment représentait $44,70 \%$ de tous les coûts de production.

Compte tenu de l'amortissement et des valeurs des coûts d'exploitation effectifs (COE), le coût total de production a été obtenu en un an (tableau 8).

Tableau 8 - Coût total de production - CTP (base annuelle) :

\begin{tabular}{|l|l|}
\hline Description & Montant total R\$ \\
\hline Dépréciation & 138,52 \\
\hline Coûts du COE & $1.615,50$ \\
\hline $\begin{array}{l}\text { Paiement } \\
\text { l'investissement }\end{array}$ & 302.37 \\
\hline Total & $\mathbf{2 . 0 5 6 , 3 9}$ \\
\hline
\end{tabular}

Source : Données du projet.

RC: 99521

Disponible en: https://www.nucleodoconhecimento.com.br/veterinaria-fr/paturage-enrotation 
Dans les pays où les prix du lait sont bas, les producteurs sont en mesure de réduire le coût de production en augmentant la participation des pâturages à l'alimentation des vaches laitières. Dans cette étude, le coût total de production représentait 28,55 $\%$ du revenu annuel.

Pour calculer les revenus, les revenus nets ont été pris en compte, c'est-à-dire la valeur gagnée dans la livraison de lait par année moins les coûts de production totaux (tableau 9).

Tableau 9 - Recettes (base annuelle) :

\begin{tabular}{|l|l|}
\hline description & Montant total R\$ \\
\hline $\begin{array}{l}\text { Revenu } \\
\text { Coût total de production(CTP sur une base } \\
\text { annuelle) }\end{array}$ & $2.200,00$ \\
\hline Balance & $\mathbf{5 . 1 4 3 , 6 1}$ \\
\hline
\end{tabular}

Source : Données du projet.

Selon Oliveira (2007), la connaissance des implications du coût opérationnel effectif (COE), à court terme, est fondamentale dans la gestion de l'entreprise, et la marge brute doit être positive, si elle est négative, l'interruption de la production est recommandée.

L'analyse financière de la mise en œuvre de ce projet s'est avérée réalisable (tableau 9). Compte tenu des analyses effectuées à partir des données obtenues, il a été observé que l'investissement dans la technique de pâturage par rotation chez les bovins laitiers présente un rendement économiquement viable du capital investi et que la même influence positive sur la production rend cette activité plus compétitive dans le secteur. Ainsi, cette technique permet l'expression potentielle de la production laitière et l'augmentation de la rentabilité des producteurs, ce qui rendrait l'activité laitière rentable, même en période de baisse du prix du lait.

RC: 99521

Disponible en: https://www.nucleodoconhecimento.com.br/veterinaria-fr/paturage-enrotation 


\section{CONSIDÉRATIONS FINALES}

Selon les résultats obtenus dans les analyses économiques, il est conclu que la mise en œuvre du pâturage en rotation et l'échange de pâturages indigènes par Brachiaria brizantha $c v$. marandu, est une gestion viable, puisque les revenus générés par la vente de lait étaient suffisants pour couvrir les coûts totaux de production.

Ce système de production semi-intensive est recommandé à la réalité locale, car il s'agit d'une alternative à l'innovation technologique, capable de réduire les dépenses liées à l'achat d'aliments et de fournir une bonne performance animale, du changement de gestion du pâturage et de l'insertion d'herbe d'une meilleure valeur nutritionnelle et d'une production de masse plus élevée.

\section{RÉFÉRENCES}

ANDRADE, Carlos Mauricio Soares de. Pastejo Rotacionado: Tecnologia para Aumentar a Produtividade de Leite e a Longevidade das Pastagens. EMBRAPA - Acre, dez. 2008.

ANUÁRIO LEITE 2019. Sua excelência, o consumidor: novos produtos e novas estratégias da cadeia do leite para ganhar competitividade e conquistar os clientes finais. Brasília, DF: Texto Comunicação Corporativa, 2019. Disponível em: $<$ https://www.infoteca.cnptia.embrapa.br/infoteca/handle/doc/1109959>. Acesso em: 25 mar. 2020.

DEMSKI, Joana Baptista. Desempenho e comportamento de vacas lactantes em pastagens de cultivares de braquiárias. Dissertação apresentada ao Programa de Pós-graduação do Instituto de Zootecnia, APTA/SAA. Nova Odessa - SP, 2013. $80 p$.

FONSECA, Delermando Miranda da; MARTUSCELLO, Janaina Azevedo. Plantas forrageiras. Viçosa, 537p. 2010.

RC: 99521

Disponible en: https://www.nucleodoconhecimento.com.br/veterinaria-fr/paturage-enrotation 
FUKUMOTO, Nelson Massaru; DAMASCENO, Júlio Cesar; DERESZ, Fermino, MARTINS, Carlos Eugênio; CÓSER, Antônio Carlos; SANTOS, Geraldo Tadeu dos. Produção e composição do leite, consumo de matéria seca e taxa de lotação em pastagens de gramíneas tropicais manejadas sob lotação rotacionada. Revista Brasileira de Zootecnia, v. 39, n.7, p. 1548-1557, 2010.

GERON, Luiz Juliano Valério; MEXIA, Alexandre Agostinho; GARCIA Jocilaine; SILVA Marciano Moreira da; ZEOULA, Lúcia Maria. Suplementação concentrada para cordeiros terminados a pasto sobre custo de produção no período da seca. Semina: Ciências Agrárias, Londrina, v. 33, p. 797-808, 2012.

GERON, Luiz Juliano Valério; MOURA, Daiane Caroline; RODRIGUES, Deivison Novaes; PAULA, Edson Júnior Heitor; TRAUTMANN-MACHADO, Raquel Joana; GARCIA, Jocilaine; SCHUMANN, Alline Mariá; SILVA; Dilma Alves. Viabilidade econômica de tourinhos terminados em confinamento alimentados com diferentes teores de caroço de algodão em dietas elaboradas com coprodutos agroindustriais. Semina: Ciências Agrárias, Londrina, v.35, p. 2673-2684, 2014.

GONÇALVES, Carlos Alberto; CAMARÃO, Ari Pinheiro; DUTRA, Saturnino; AZEVEDO, Guilherme Pantoja Calandrini de; MENDONÇA, Denise Castro; SOBRINHO, Carlos de Melo Junior. Produção de leite em pastejo rotacionado intensivo de "Brachiaria brizantha" cv. Marandu sob dois níveis de suplementação concentrado. In: Reunião anual da sociedade brasileira de zootecnia, 40., 2003, Santa Maria. Anais... Santa Maria: Sociedade Brasileira de Zootecnia, 2003.

IBGE -Instituto Brasileiro de Geografia e Estatística. [2018]. Pesquisa da pecuária nacional disponível em: <https://sidra.ibge.gov.br/Tabela/74>. Acessado em: 25 mar. 2020.

RC: 99521

Disponible en: https://www.nucleodoconhecimento.com.br/veterinaria-fr/paturage-enrotation 
IPARDES: Instituto Paranaense de Desenvolvimento Econômico e Social. Caracterização sócio econômica da atividade leiteira no Paraná: Sumário executivo. Curitiba: IPARDES, 2009. 29 p.

JANK, Liana; ANDRADE, Carlos Mauricio Soares de; BARBOSA, Rodrigo Amorim; MACEDO, Manuel Claudio Motta; VALERIO, Jose Raul; VERZIGNASSI, Jaqueline Rosemeire; ZIMMER, Ademir Hugo; FERNANDES, Celso Dornelas; SANTOS, Mateus Figueiredo; RESENDE, Rosangela Maria Simeão. O capim-BRS Quênia (Panicum maximum Jacq.) la diversification et l'intensification des pâturages. Disponible en: <https: ainfo.cnptia.embrapa.br/digital/bitstream/item/165106/1/capimbrs-quenia-panicum-maximum-jacq.pdf="'">.</https:> Accès le: 25 Mar. 2020.

MARION, Jose Carlos; SEGATTI, Sonia. Contabilidade da Pecuária. 9 ed. São Paulo: Atlas, 2010.

MARTIN, Nelson Batista; SERRA, Renata; OLIVEIRA, Marli Dias Mascarenhas; ÂNGELO, José Alberto; OKAWA, Hiroshige. Sistema "CUSTAGRI": sistema integrado de custos agropecuários. São Paulo: IEA/SAA, 1997. p. 1-75.

MARTINEZ, Junior Cesar. Produção de leite a pasto: manejo do pastejo é fundamental para manter a produção. 2009. Disponível em: $<$ http://www.milkpoint.com.br/radartecnico/nutricao/producao-deleite-a-pasto-manejodo-pastejo-e-fundamental-para-manter-aproducao-51710n.aspx>. Acesso em: 27 mai. 2020.

MATSUNAGA, Minoru; BEMELMANS, Paul Frans; TOLEDO, Paulo Edgard Nascimento de; DULLEY, Richard Domingues; HIROSHIGE, Okawa; PEDROSO, Iby Arvatti. Metodologia de custo utilizada pelo IEA. Agricultura em São Paulo, v. 23, n. 1, p.123-39, 1976.

OLIVEIRA, Jefferson Soares de. Análise de eficiência e alocação de recursos na produção leiteira do estado do Rio de Janeiro. In: congresso da sociedade

RC: 99521

Disponible en: https://www.nucleodoconhecimento.com.br/veterinaria-fr/paturage-enrotation 
brasileira de economia, administração e sociologia rural, 45., 2007. Londrina. Conhecimentos para a agricultura do futuro: Anais... Brasília, DF: SOBER; Londrina: IAPAR: Universidade Estadual de Londrina, 2007.

PEREIRA, Lilian Elgalise Techio. et al. Recomendações para correção e adubação de pastagens tropicais. Pirassununga: Faculdade de Zootecnia e Engenharia de Alimentos da USP, 2018. 56p.

ROSESTOLATO, Lucas Luiz Rocha. et al. Viabilidade econômica comparativa de sistemas tecnológicos da pecuária leiteira no município de llha Solteira/SP. Congresso de extensão universitária da UNESP, 8., p. 1-6, 2015. Disponível em: <http://hdl.handle.net/11449/142491>. Acesso em: 28 mai. de 2020.

SEAB - Secretaria de Estado da Agricultura e do Abastecimento. Bovinocultura de leite: leite - $\quad$ produção 2016/17. $\quad$ Disponível em:<http://www.agricultura.pr.gov.br/arquivos/File/deral/Prognosticos/2018/leite_201 7_18.pdf>. Acesso em: 25 mar. de 2020.

Publié : Juillet 2021.

Approuvé : Septembre 2021.

RC: 99521

Disponible en: https://www.nucleodoconhecimento.com.br/veterinaria-fr/paturage-enrotation 